\title{
Echocardiographic demonstration of important abnormalities of the mitral valve in congenitally corrected transposition
}

\author{
Daniel J Penny, Jane Somerville, Andrew N Redington
}

\begin{abstract}
Whereas abnormalities of the morphological tricuspid valve are common in the setting of discordant atrioventricular connections, there are only a few postmortem reports of abnormalities of the mitral valve in this condition. This report describes two patients with discordant atrioventricular connections, in whom important abnormalities of the mitral valve were found during life by cross sectional echocardiography.
\end{abstract}

(Br Heart J 1992;68:498-500)

There are few reports of abnormalities of the morphological mitral valve in patients with congenitally corrected transposition of the great arteries. Furthermore, it has been suggested that when present, these abnormalities are likely to be clinically unimportant. ${ }^{1} \mathrm{We}$ have recently seen two patients with congenitally corrected transposition of the great arteries, both of whom were found to have important abnormalities of the mitral valve. It is unusual for these abnormalities to be diagnosed in life by cross sectional echocardiography.

\section{Case reports}

PATIENT 1

A four year old girl was referred to our hospital with a diagnosis of congenitally corrected transposition of the great arteries, mild pulmonary stenosis, and left atrioventricular valve (tricuspid valve) regurgitation. She remained well until epigastric pain and hepatomegaly developed when she was 13 years old.

A transthoracic echocardiogram performed at that time confirmed the presence of the usual atrial arrangement, with discordant atrioventricular and ventriculoarterial connections. The only haemodynamically important additional finding was of severe obstruction of the left ventricular outflow tract related to an aneurysm of the mitral valve that had prolapsed into the subpulmonary region (fig 1A). Transoesophageal echocardiography confirmed the presence of a large aneurysm of the anterior leaflet of the mitral valve (fig 1B). Cardiac catheterisation showed a peak systolic gradient of $100 \mathrm{~mm} \mathrm{Hg}$ across the left ventricular outflow tract and a large aneurysm of the mitral valve was seen prolapsing into the pulmonary outflow tract during ventriculography (fig 2).
PATIENT 2

An 18 year old man was referred for assessment of complex congenital heart disease. He had developed cyanosis in the neonatal period and underwent cardiac catheterisation in another institution, at which a diagnosis of usual atrial arrangement, absent right atrioventricular connection, and a discordant ventriculoarterial connection was formed. There was progressive pulmonary stenosis with reduced pulmonary blood flow and so a right Blalock-Taussig shunt was performed when he was six years old. He was admitted to this hospital aged 23 years with a view to planning further management.

Cross sectional echocardiography unexpectedly showed the usual atrial arrangement, a discordant atrioventricular connection, and a discordant ventriculoarterial connection. There was a large ventricular septal defect with subpulmonary and valvar pulmonary stenosis. The left ventricle was diminutive and colour Doppler echocardiography failed to identify any flow across the miniature mitral valve (fig 3). The patient subsequently had an uncomplicated Fontan operation (atriopulmonary anastomosis). At surgery a pinhole orifice was detected in the mitral valve; this was closed by direct suture.

\section{Discussion}

These patients show abnormalities of the morphological mitral valve in congenitally corrected transposition of the great arteries. We are not the first to show the presence of such abnormalities in this group of patients. They were also found in $55 \%$ of specimens studied at necropsy by Gerlis and co-workers. ${ }^{1}$ It has been suggested, however, that such abnormalities tend to be minor and of doubtful surgical significance. The development of subpulmonary stenosis in patient 1 , related to an aneurysm of the mitral valve, highlights the intimate relation between the pulmonary outflow tract and the atrioventricular valves in hearts with a discordant ventriculoarterial connection, which causes the pulmonary valve to be wedged between the mitral and tricuspid orifices.

The development of important obstruction of the pulmonary outflow tract is a common complication of congenitally corrected transposition of the great arteries, occurring in about $40 \%$ of patients. ${ }^{2}$ This obstruction may be valvar, muscular or, more usually, related to aneurysms of the septal structures and tissue tags blocking the outflow tract. It is imperative 

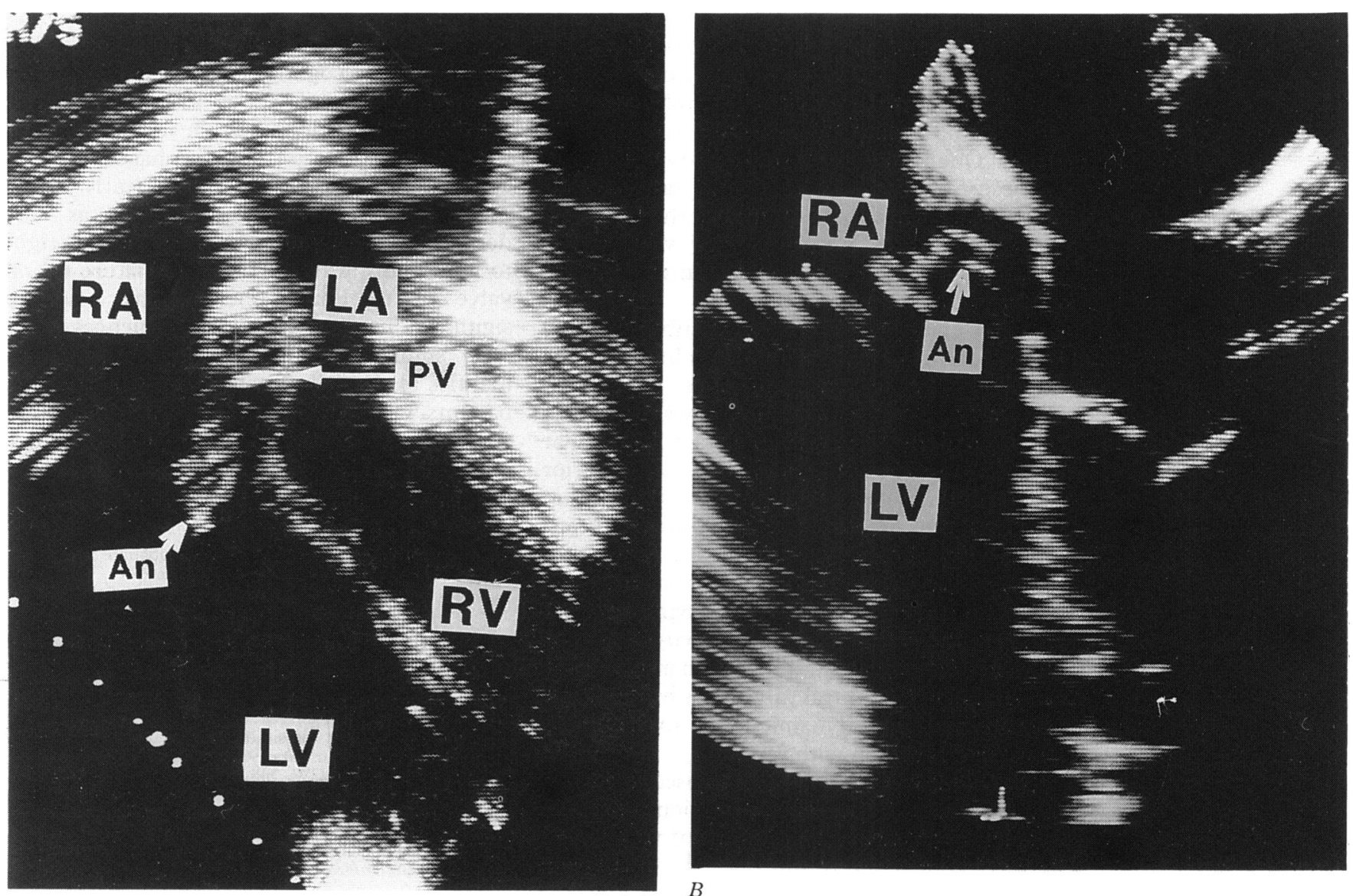

$B$

A

Figure 1 (A) Transthoracic echocardiogram, taken during ventricular diastole, from an apical window in patient 1. There are discordant atrioventricular connections. The mitral and pulmonary valves (PV) are in fibrous continuity and there is an aneurysm on the mitral valve $($ An). (B) Transoesophageal echocardiogram in patient 1 during ventricular systole. The aneurysm on the mitral valve is prolapsing towards the pulmonary outflow tract. $R A$, right atrium; $L A$, left atrium; $L V$, left ventricle; $R V$, right ventricle.

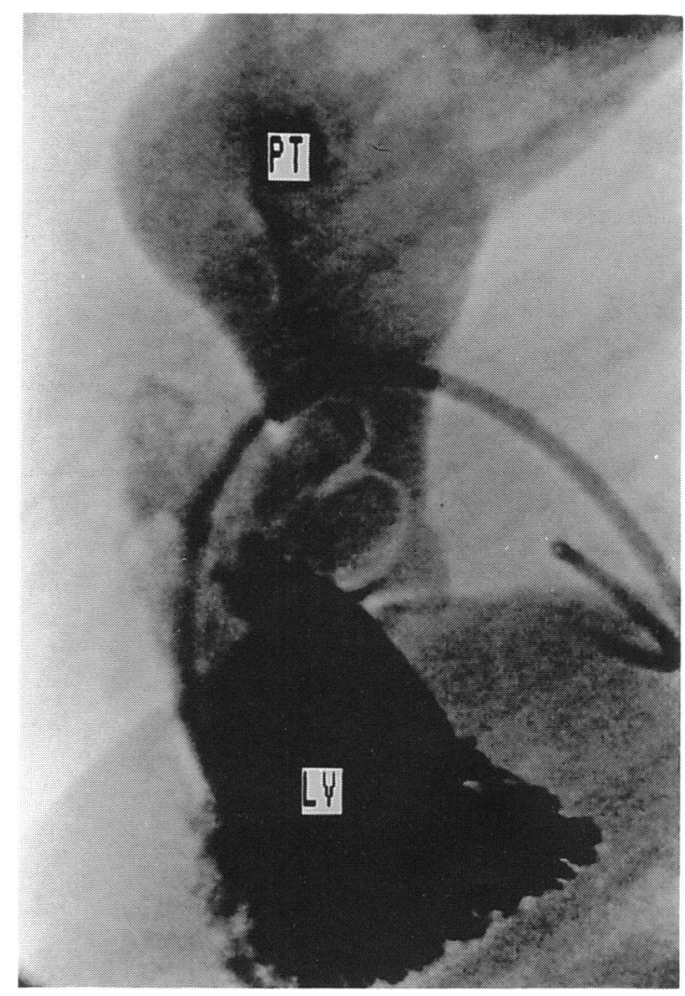

Figure 2 Lateral projection of a left ventricular angiogram in patient 1 , shows prolapse of the aneurysms on the mitral valve into the pulmonary outflow tract causing pulmonary obstruction. $L V$, left ventricle; $P T$, pulmonary trunk.

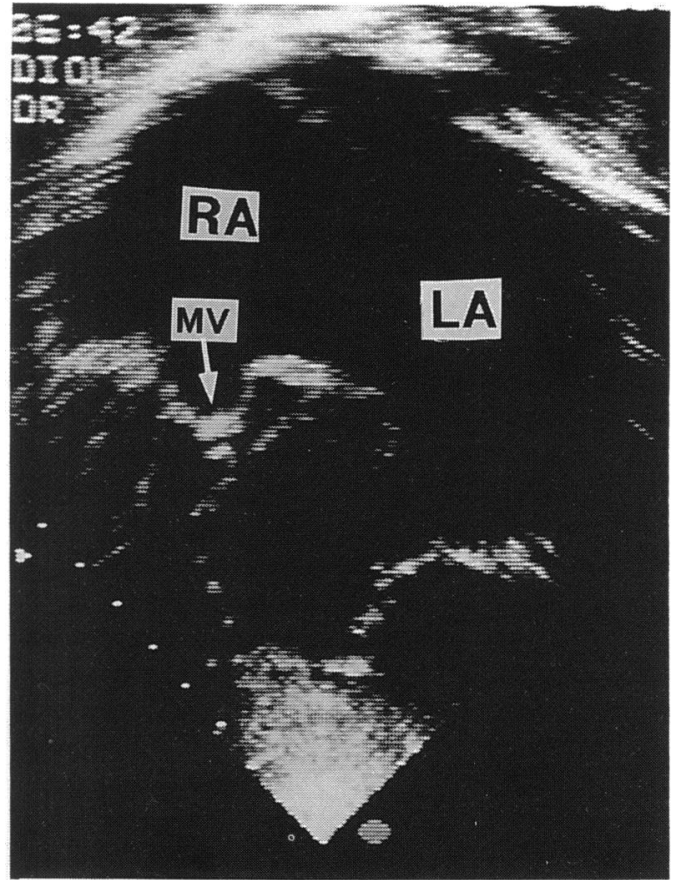

Figure 3 Transthoracic echocardiogram in patient 2 shows a miniaturised mitral valve (MV). Although appearing imperforate, a patent but severely stenosed orifice was found at surgery. $R A$, right atrium; $L A$, left atrium. 
to identify accurately the origin of these aneurysms and tags when surgical resection is contemplated, because of the close relation between the atrioventricular conduction axis and the pulmonary outflow tract in this condition. ${ }^{2}$ One pathological study showed that the source of these aneurysms and tags may be from the membranous septum, the tricuspid valve, or from the pulmonary valve itself, although this study did not identify a mitral valve origin in any case. ${ }^{2}$

Levy and co-workers showed subpulmonary obstruction related to abnormalities of the mitral valve in three patients studied at necropsy. ${ }^{3}$ One of these patients was similar to ours, in that there was an intact ventricular septum and a $2 \mathrm{~cm}$ aneurysm of the anterior leaflet of the mitral valve, which prolapsed into the left ventricular outflow tract. To our knowledge, however, ours is the first antemortem report of this phenomenon. Interestingly, in retrospect, an echocardiogram performed on the patient of Levy and co-workers at the age of four years had shown an insignificant aneurysm of the mitral valve, thus highlighting the ability of this dynamic lesion to change its form and function with time. ${ }^{14}$

Patient 2 was originally diagnosed as having no right atrioventricular connection, a dominant left ventricle, and a discordant ventriculoarterial connection. It must be emphasised that this diagnosis was made before the introduction of cross sectional echocardiography. The subsequent recognition of a biventricular atrioventricular connection when he was seen in this hospital emphasises the need for careful transthoracic or transoesophageal echocardiograms in these patients. Clearly it had important surgical implications, as the development of regurgitation or diastolic right to left shunting through the miniature mitral valve after the Fontan operation may have had significant haemodynamic consequences.

In summary, clinically important abnormalities of the mitral valve may be present in patients with congenitally corrected transposition of the great arteries. These abnormalities may be readily identified by careful cross sectional echocardiography.

DJP is supported by the British Heart Foundation.

1 Gerlis LM, Wilson N, Dickinson DF. Abnormalities of the mitral valve in congenitally corrected transposition (discordant atrioventricular and ventriculoarterial connections). Br Heart J 1986;55:475-9.

2 Anderson RH, Becker AE, Gerlis LM. The pulmonar outflow tract in classically corrected transposition. Thorac Cardiovasc Surg 1975;69:747-57.

3 Levy MJ, Lillihei CW, Elliott LP, Carey LS, Adams P, Edwards JE. Accessory valvular tissue causing subpulmonary stenosis in corrected transposition of great vessels. Circulation 1963;27:494-502.

4 Somerville J. Congenital heart disease-changes in form and function. Br Heart Journal 1979;41:1-22. 